\title{
Misconceptions About Incline Speed for Nonlinear Slopes
}

\author{
Doug Rohrer \\ University of South Florida
}

\begin{abstract}
In 3 experiments, college students provided qualitative predictions about a marble's speed along nonlinear inclines. When predicting the outcome of a race between identical marbles along differently shaped ramps, most students predicted incorrectly that the shorter path was necessarily quicker (the shorter-quicker belief). When comparing instantaneous speed at 2 points, most students predicted incorrectly that incline speed depended on the slope at that point (the slope-speed belief). A final experiment provides evidence that the slope-speed belief reflects a deeper fallacy regarding the resistance encountered while traversing inclines and lifting objects. This fallacy also predicts the prevalent belief that heavier objects fall faster than lighter objects during incline descent or free fall.
\end{abstract}

Despite a lifetime of experience on the earth's surface, many people hold strikingly false beliefs about the qualitative effects of gravity on rolling, falling, and sliding. Previous findings have revealed that these beliefs sometimes reflect deeper misconceptions, and that the nature of these misconceptions can provide insight into how people come to understand and misunderstand the laws that govern the effects of gravity on motion. These studies have examined a wide range of phenomena, including pendulum motion (e.g., Pittenger, 1985), the trajectories of thrown objects (e.g., Hecht \& Bertamini, 2000; Krist, Fieberg, \& Wilkening, 1993; McBeath, Shaffer, \& Kaiser, 1995), the speed of free fall (e.g., Shanon, 1976), incline motion (e.g., Hecht, 1993; Proffitt, Kaiser, \& Whelan, 1990), and the perception of inclines (Bhalla \& Proffitt, 1999; Creem \& Proffitt, 1998; Proffitt, Bhalla, Gossweiler, \& Midgett, 1995). The experiments reported here concern people's understanding of incline speed as a function of incline shape, and the data reveal two false beliefs that are at odds with everyday motion. Their description is preceded by a brief review of the relevant Newtonian theory.

\section{Incline Speed}

The effect of incline shape on incline speed is remarkably simple. At any point along an incline, an object's speed depends on the net vertical drop at that point, regardless of the ramp shape prior to that point. Mathematically, incline speed equals $(\mathrm{kgh})^{1 / 2}$, where $h$ represents the object's net vertical drop since its release, $g$ equals the rate of gravitational acceleration on the earth's surface $\left(9.8 \mathrm{~m} / \mathrm{s}^{2}\right)$, and $k$ depends on the object's shape and its distribution of mass (see, e.g., Halliday \& Resnick, 1981). This study relies on uniformly dense spheres, for which $k=10 / 7$ (e.g., Halliday \& Resnick, 1981). Because $k$ depends solely on the object rather than

I thank Don Helling and Eric Ruthruff for their insights; Eric-Paul Bomgren, Danielle Mazur, Rena Mehta, and Elizabeth Ocampo for their assistance with data collection; Mukul Bhalla, John Pittenger, and an anonymous reviewer for their comments; and J. David Rohrer for his carpentry skills.

Correspondence concerning this article should be addressed to Doug Rohrer, Department of Psychology, University of South Florida, PCD 4118G, Tampa, Florida 33620-7800. E-mail: drohrer@chuma1.cas.usf.edu the ramp, it remains constant in scenarios involving identical objects and varying ramp shapes, as in the experiments reported here. Therefore, for a given object on the surface of the earth, incline speed varies with vertical drop.

Incline speed can also be affected by friction, but this complication is not problematic in this study for two primary reasons. First, the present experiments required qualitative comparisons rather than quantitative estimates, and the quantities under comparison were each subject to friction. Hence, the selection of the correct answer did not depend on whether friction was neglected. Second, the impeding effect of friction on rolling speed is relatively small, demonstrated in that a roller coaster can freely roll to the top of a hill that is nearly as high as the previous hill.

\section{The Shorter-Quicker Belief}

Because incline speed depends on vertical drop, the average speed along a linear ramp between two points of unequal elevation is slower than the average speed along a concave ramp adjoining the same two points (as in Figure 1A). Hence, the greater initial steepness of a concave ramp allows the marble to quickly descend to points of lower elevation where speed is greater. In fact, the quickest descent between any two points of unequal elevation is a concave ramp, because the greater vertical drop produces more than enough speed to compensate for its longer distance (in relation to the linear ramp). The shape of the curve in Figure 1A happens to be the quickest shape, in fact: It is the curve of the brachistochrone (shortest time). It is one half of an inverted cycloid, which is defined as the path traced by a point on the rim of a wheel rolling along a horizontal surface (cf. Anton, 1980; Rohrer, 1994). The precise form of the brachistochrone curve was unknown until Johann Bernoulli and Wilhelm Leibniz presented the problem as a challenge in 1696 (Anton, 1980; Bell, 1937). Several mathematicians solved the problem shortly thereafter, and Isaac Newton reportedly solved the problem in one evening (Bell, 1937). Incidentally, the inverted cycloid is also the curve of the tautochrone (equal time), meaning that an object can be released from any point along this curve without affecting the duration of the descent (e.g., Anton, 1980). 


\section{A Slope-Speed Belief}

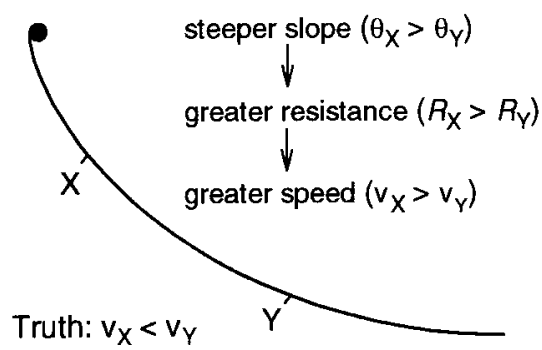

\section{B Mass-Speed Belief}

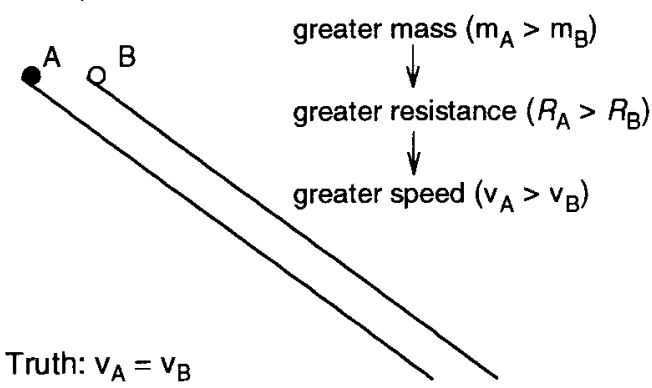

Figure 1. Predictions of the resistance-speed fallacy. A: The slope-speed belief-Slope is greater at Point $\mathrm{X}$ than at Point $\mathrm{Y}\left(\theta_{\mathrm{X}}>\theta_{\mathrm{Y}}\right)$, and an object held at Point $\mathrm{X}$ therefore provides greater resistance $\left(R_{\mathrm{X}}>R_{\mathrm{Y}}\right)$; thus, the resistance-speed fallacy predicts greater speed at the steeper point $\left(v_{\mathrm{X}}>\right.$ $\left.v_{\mathrm{Y}}\right)$. However, in truth, speed is greater at the point with greater vertical drop $\left(v_{\mathrm{X}}<v_{\mathrm{Y}}\right)$. B: The mass-speed belief-Mass is greater for Marble A than for Marble $\mathrm{B}\left(m_{\mathrm{A}}>m_{\mathrm{B}}\right)$, and resistance is therefore greater for Marble A when one attempts to push either marble uphill or hold it in place $\left(R_{\mathrm{A}}>R_{\mathrm{B}}\right)$; hence, the resistance-speed fallacy predicts greater speed for Marble A $\left(v_{\mathrm{A}}>v_{\mathrm{B}}\right)$. However, the marbles' speeds are actually equal $\left(v_{\mathrm{A}}=v_{\mathrm{B}}\right)$.

It is perhaps surprising that a linear descent is not the quickest, because there is intuitive appeal to the notion that the shortest path should be the quickest. This view is dubbed the shorter-quicker belief, and its prevalence is assessed in Experiment 1. This experiment required participants to predict the outcome of a race between two identical marbles along differently shaped ramps that started at the same point and finished at the same point. The experiment used a wooden apparatus; the shape and relative position of the ramps is illustrated in Figure 2A. The ramps descended along an identical arc before diverging, and the latter portion of each ramp included either a dip or a hill. The dip ramp shape appears in Figure 2B; the hill is simply the mirror image of the dip (see Appendix A). While viewing this apparatus, participants were asked to choose the quicker of the two ramps, as described in Figure 3A. The dip ramp is the quicker ramp, because the net vertical drop is greater along the dip than along the hill. In fact, speed at every point within the dip exceeds the speed at every point along the hill, and the margin of victory is large.

\section{The Slope-Speed Belief}

During informal debriefings with college students who answered the race question shown in Figure $3 \mathrm{~A}$ as part of a pilot questionnaire, several expressed a fundamental misunderstanding about incline speed. Specifically, some defended their prediction of a simultaneous finish by explaining that both the dip and the hill include one "fast" downhill and one "slow" uphill. Hence, whereas speed actually depends on vertical drop, these students attributed incline speed to slope.

This misguided view is one instantiation of what is dubbed here the slope-speed belief, and it attributes an object's incline speed at a point to the incline slope at that point. In particular, the slopespeed belief specifies that incline speed is faster at a point along a steep section than at a point on a more gradual section. Likewise, the slope-speed belief holds that incline speed is greater at "downhill points" than at "flat points," and in turn, greater at "flat points" than at "uphill points." The slope-speed belief is sharply inconsistent with Newtonian theory, as illustrated by an object's speed along a concave ramp like that in Figure 1A. In this case, the slope-speed belief predicts continuously decreasing speed, when in fact, speed continuously increases.

The slope-speed belief is refuted by many real-world experiences. The speed of a roller coaster is slower when beginning to travel downward than it is shortly after it begins climbing up a following hill. Likewise, a skateboarder who rolls back and forth

\section{A The Ramps}

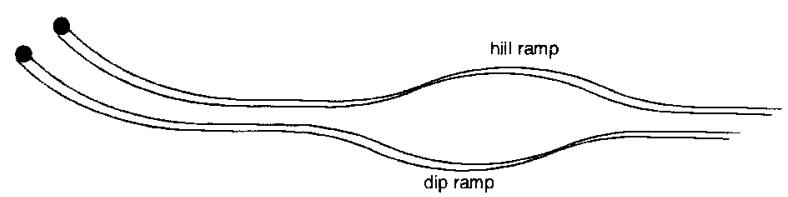

8 Two-Dimensional Plot of Basic Ramp Shape

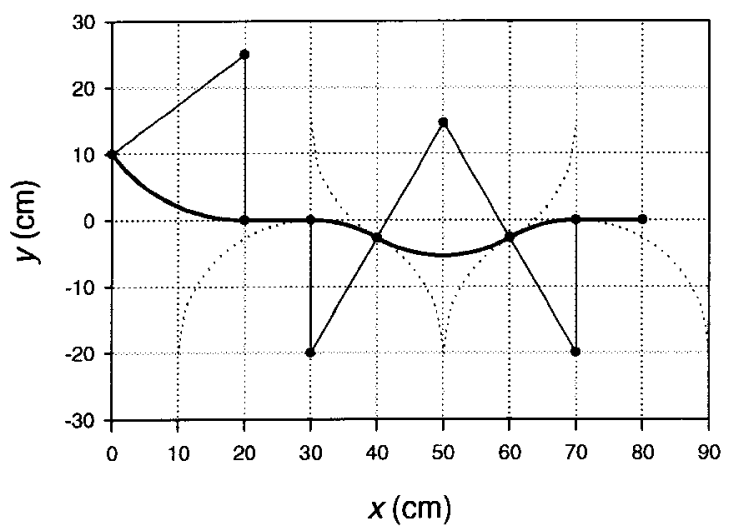

Figure 2. A: The ramps used in Experiment 1. B: The shape of the ramps. Both were composed entirely of arcs and line segments (see Appendix A). 


\section{A Dip-Hill Race Question (brief version) Marbles $A$ and $B$ are identical. The marbles are released simultaneously. Which marble wins, if any? \\ * Marble A (on the dip ramp) \\ * Marble B (on the hill ramp) \\ * The marbles finish at virtually the same instant}
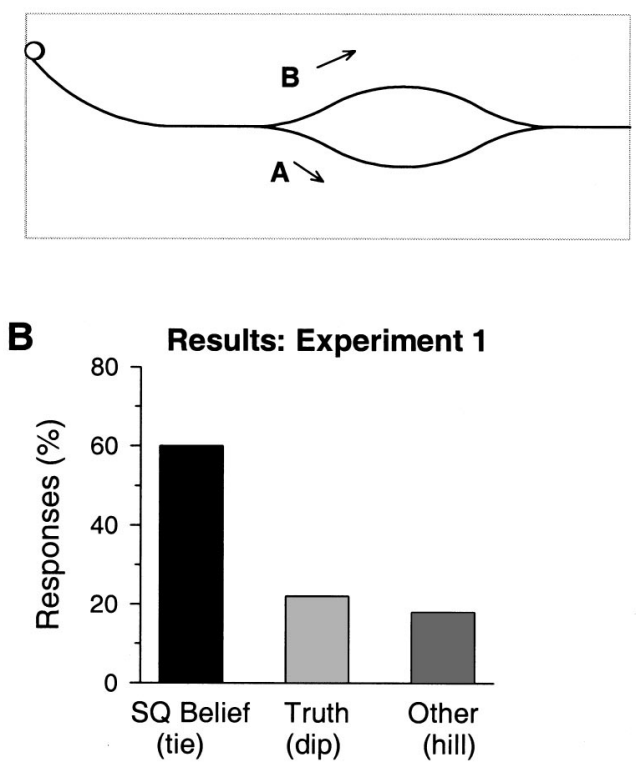

Figure 3. A: The race question. The dip ramp is quicker, but the shorterquicker (SQ) belief predicts a tie. B: Results of Experiment 1.

along a U-shaped ramp reaches maximum speed along the bottom, although the ramp is steeper near the top. In addition, certain brands of toy cars and so-called derby cars are commonly rolled along ramps with slopes that are initially steep and subsequently more gradual, and the cars' speeds increase as the ramps' slopes flatten. And the same is true for the motion of sleds and skiers.

The appeal of the slope-speed belief was assessed in Experiment 2 by use of the speed question shown in Figure 4A. This task required a comparison of speed at a point on one ramp to the speed at a point on a second ramp. Because the two ramps began at the same height, speed was greater at the point with lower elevation.

\section{The Resistance-Speed Fallacy}

The slope-speed belief may reflect a deeper misconception that attributes incline speed to the resistance encountered as people move objects or their bodies along inclines. When pushing an object uphill or merely holding an object in place at a point along a hill, one must exert an amount of force that depends on the object's resistance to his or her efforts. This resistance reflects the force that propels the object downward, and the magnitude of this resistance is greater at steeper slopes. Formally, the resisting force equals the vector sum of the gravitational force and the so-called normal force. These concepts are described fully in Appendix B.

Although most people possess no formal understanding of this law relating slope and resistance, everyone is very aware of its implications. When pushing a wheelbarrow uphill, for instance, one must exert greater effort at points of steeper slope. Likewise, it is harder to climb a steep hill than a more gradual one. Of course, gravity facilitates people's efforts during descents, but they must nevertheless control the speed of these descents by exerting an amount of force that varies with the downward force. Hence, regardless of whether one is moving an object uphill, downhill, or simply holding it in place at a point along an incline, she or he must exert a force that is modulated in response to the encountered resistance. Moreover, the resistance is greater along steeper as-

\section{A Speed Question (brief version) Marbles $A$ and $B$ are identical. Regardless of how quickly either marble reaches any particular point, which marble, if any, is rolling faster at the instant it reaches the vertical line? \\ * Marble A \\ * Marble B \\ * Equal}
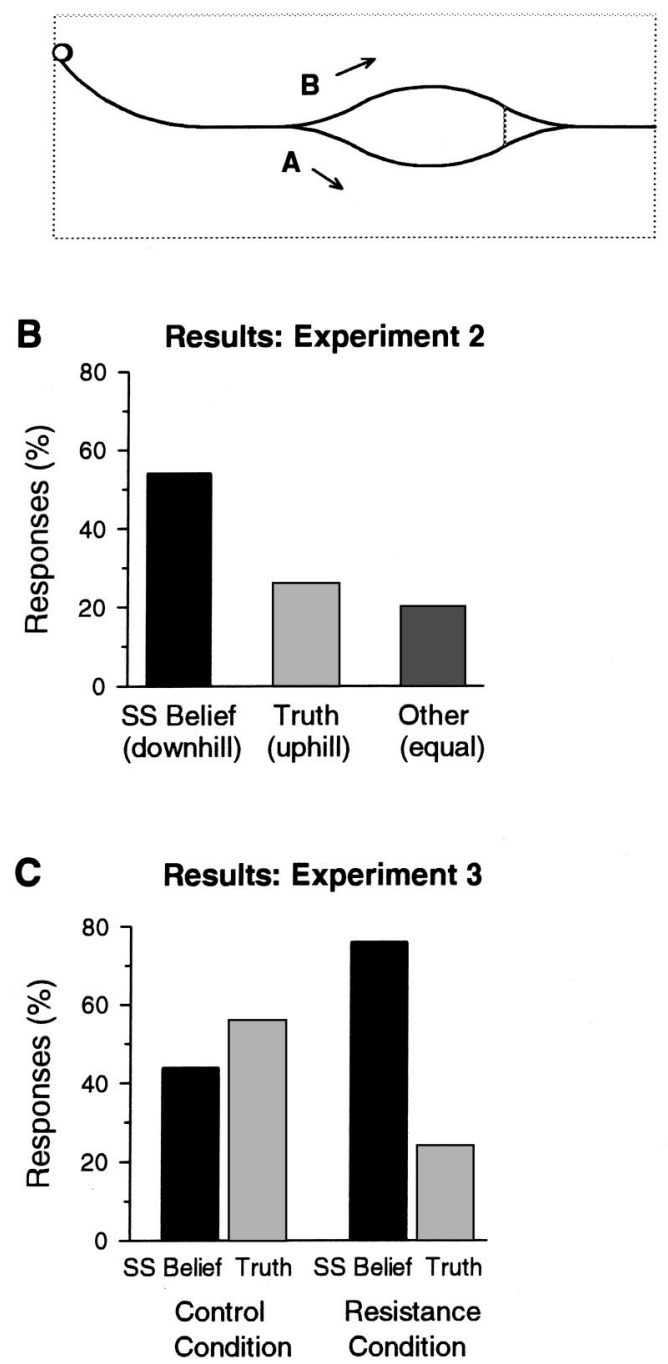

Figure 4. A: The speed question. Speed is greater at the point with lower elevation, but the slope-speed (SS) belief predicts greater speed at the downhill point. B: Results of Experiment 2. C: Results of Experiment 3. In this experiment, participants were presented with only two response options: the slope-speed response ("downhill point") and the correct answer ("uphill point"). 
cents than along more gradual ascents, and the resistance during ascents is less than that during descents.

Therefore, if people conclude falsely that the magnitude of this resistance predicts an object's incline speed after it is released, the slope-speed belief follows logically. This misguided view is termed the resistance-speed fallacy, and it explains the slopespeed belief as follows: If slope predicts resistance and resistance predicts speed, then slope predicts speed. The derivation is also illustrated in Figure 1A. In the figure, the marble descends a concave incline that has two points indicated. The encountered resistance is greater at the "steeper point," and the resistancespeed fallacy therefore predicts greater speed at this "steeper point." Yet speed is actually greater at the "gradual point," because it lies at a lower elevation.

The resistance-speed fallacy holds intuitive appeal, because it seems reasonable that the encountered resistance would predict the object's speed on release. In fact, encountered resistance does predict speed in other scenarios. In archery, for instance, the resistance encountered before releasing the bow string predicts the arrow's speed after release. Likewise, the magnitude of encountered resistance predicts the subsequent speed of slingshots and catapults. In a similar manner, the greater resistance at steeper slopes is akin to the greater resistance of a tighter rubber band. With the resistance-speed fallacy, then, pushing an object uphill is similar to stretching a spring or rubber band: The object snaps back toward the earth with speed that depends on the resistance. This resistance also depends on the object's mass, of course. The next section describes how the resistance-speed fallacy also predicts false beliefs concerning the effect of mass on free fall and free roll.

\section{The Mass-Speed Beliefs}

Perhaps the most oft-cited misconception is the widely held belief that heavier objects fall to the ground more quickly than lighter objects. This belief is not entirely false, however, because greater mass can increase the speed of free fall in the presence of air resistance. For instance, although a feather falls as quickly as a rock in a vacuum, the feather falls much slower than the rock in the real world. Hence, the rock's greater mass provides some immunity to the impeding effects of air resistance.

In many scenarios, however, an object's mass has no visibly discernable effect on its falling speed. For instance, if two stones of equal size and unequal mass are dropped simultaneously, the stones land at nearly the same instant. Students are taught perennially that Galileo conducted this very experiment, although scholars agree that he did not drop the stones from atop the Tower of Pisa (Crawford, 1996). Despite this null effect of mass, a striking number of people falsely believe that the heavier stone falls more quickly than the lighter stone.

In a similar manner, many people appear to believe that heavier objects descend inclines more quickly than lighter objects, when, in fact, an object's mass has no discernable effect on its incline speed. In a study by Proffitt et al. (1990), more than one half of a college-student sample predicted incorrectly that a heavier object would roll down a linear incline at a greater speed than a lighter object of the same size and shape. In summary, there appears to be broad support for the belief that greater mass yields greater speed during both free fall and free roll. These two beliefs are termed here the mass-speed beliefs. The two beliefs are obviously similar, and in fact, Galileo derived his findings on free fall by observing incline motion (Crawford, 1996; Drake, 1989).

In addition to the slope-speed beliefs, the mass-speed beliefs are predicted by the resistance-speed fallacy. In particular, the resistance encountered while pushing an object along an incline or lifting it from the ground reflects the gravitational force propelling it downward, and the magnitude of this gravitational force varies directly with the object's mass, in accordance with Newton's law of universal gravitation (see, e.g., Halliday \& Resnick, 1981). Hence, because mass predicts resistance, an individual who falsely believes that resistance predicts speed should logically conclude that mass predicts speed. This argument is illustrated in Figure 1B. In the figure, the two marbles have different mass and are therefore propelled downward by unequal force. Consequently, if the marbles are pushed uphill or merely held in place, the more massive marble offers greater resistance. Therefore, the resistance-speed fallacy falsely predicts a quicker descent for the more massive object, when, in actuality, the objects finish simultaneously.

This account of the mass-speed beliefs is parsimonious, because it reflects people's interaction with an object and the very salient relationship between mass and resistance. For instance, before two stones of unequal mass can be dropped, the stones must first be lifted and held aloft. During this phase, the heavier stone seeks the earth's surface with greater force. By the metaphor invoked in the previous section, the lifting of a stone is akin to the stretching of a rubber band. Just as a tight rubber band snaps back to its original size more quickly than a loose rubber band, the resistance-speed fallacy holds that the heavier stone should snap back to the ground with greater speed than the lighter stone.

\section{Overview of the Experiments}

Experiment 1 used a race question to measure the appeal of the shorter-quicker belief, and Experiment 2 used a speed question to assess the prevalence of the slope-speed belief. Experiment 3 assessed the possibility that the slope-speed belief reflects the resistance-speed fallacy.

\section{Experiment 1}

Participants in this experiment answered the race question shown in Figure 3A. As described in the introduction, the dip ramp is quicker, because the dip lies entirely beneath the hill ramp. In contrast, the shorter-quicker belief predicts a tie, because the ramps are equally short.

\section{Method}

Participants. A total of 50 undergraduate students from the University of South Florida participated in return for course credit. This sample included 36 women and 14 men; 28 reported that they had completed at least one physics course in high school or college.

Materials and procedure. The apparatus is depicted in Figure 2, and its precise specifications are given in Appendix A. Each ramp was constructed by cutting along the edges of two 5-mm thick boards, and the boards were separated by a 7-mm gap that provided the track for the marble. The marbles were uniformly dense metal spheres with diameters of approximately $1 \mathrm{~cm}$. For the first experiment, the dip ramp and the hill ramp were labeled $A$ and $B$, respectively. Participants were tested in groups.

I read the following question aloud (and performed the actions in parentheses): "These two marbles are identical (I showed both marbles to 
participants). Marble A is placed at the start of the ramp with the dip (I placed the marble at the start of the dip ramp and pointed to the dip). Marble B is placed at the start of the ramp with the hill (I placed the second marble at the start of the hill ramp and pointed to the hill). Both marbles will be released at the same instant. Which marble will win, or will the two marbles finish at virtually the same moment? Take your time, and when you're ready, circle your answer." Participants' answer sheets included three alternatives: Marble $A$ wins the race, Marble $B$ wins the race, and The marbles finish at virtually the same moment. After answering the question, participants wrote brief explanations for their responses.

\section{Results and Discussion}

As shown in Figure 3B, most participants chose the shorterquicker response. In particular, $60 \%$ predicted incorrectly that the marbles would finish at virtually the same moment. A binomial test of proportions revealed this percentage to be statistically significant $(z=4.00, p<.01)$. Only $22 \%$ predicted correctly that the dip ramp was quicker, and $18 \%$ chose the hill ramp.

The popularity of the shorter-quicker response was unaffected by either gender or the completion of a prior physics course. The response percentages for the relevant demographic groups are listed in Appendix C. A majority of each group selected the shorter-quicker response (all $z \mathrm{~s}>2.47$, all $p \mathrm{~s}<.01$ ). In addition, a chi-square test of independence revealed that the likelihood of choosing the belief response was independent of both gender, $\chi^{2}(1)=2.79, n s$, and the completion of a prior physics course, $\chi^{2}(1)=0.14, n s$. Likewise, the number of prior physics courses was not significantly correlated with the likelihood of choosing the shorter-quicker response $\left(r_{\mathrm{pb}}=.05, n s\right)$.

The participants' reasons supported their answers. The relative frequency of these reasons is listed in Table D1 of Appendix D. Of those participants who falsely predicted the shorter-quicker response of tie, $77 \%$ effectively cited the shorter-quicker belief by citing the ramps' equal length or symmetry (which logically ensures their equal lengths). Of those who correctly predicted that the dip ramp was quicker, 55\% argued appropriately that the dip increases speed (or that the hill slows speed), and 36\% noted more ambiguously that the dip's downhill part increases speed. Finally, of those who incorrectly predicted that the hill ramp was quicker, $44 \%$ believed that the hill was quicker because its uphill portion preceded its downhill portion. In summary, the results of Experiment 1 provide evidence for the prevalence of the shorter-quicker belief, and its appeal appears to be unaffected by gender or prior physics instruction.

\section{Experiment 2}

Participants in this experiment answered the speed question that is shown in Figure 4A, which assessed the appeal of the slopespeed belief. As described in the introduction, the speed question is answered correctly by simply choosing the point with lower elevation. Yet the slope-speed belief holds that speed is greater at the point of higher elevation, because this point is located on a downhill slope.

\section{Method}

Participants. A total of 50 undergraduate students from the University of South Florida participated in return for course credit. This sample included 40 women and 10 men; 22 reported that they had completed at least one physics course in high school or college. None had participated in Experiment 1.

Materials and procedure. This experiment used the same apparatus and procedure as in the previous experiment, but the question was different. The question was read aloud as follows (and actions in parentheses were performed): "These two marbles are identical (I showed the marbles to participants). Marble A is placed at the start of the ramp with the dip (I placed the marble at the start of the dip ramp and pointed to the dip). Marble B is placed at the start of the ramp with the hill (I placed a second marble at the start of the hill ramp and pointed to the hill). But the marbles will not be released at the same time. Instead, the marbles will be released one at a time. Predict which marble will be rolling faster at the instant it reaches the indicated mark." Participants' answer sheets included three alternatives: Marble A is faster, Marble B is faster, and The marbles' speeds are equal. After answering the question, participants wrote brief explanations for their responses.

\section{Results and Discussion}

As shown in Figure 4B, most participants chose the slope-speed response. Specifically, $54 \%$ predicted greater speed at the "downhill point" than at the "uphill point." A binomial test of proportions revealed this percentage to be statistically greater than chance $(z=$ $3.10, p<.01$ ). Just $26 \%$ predicted correctly that the incline speed was greater at the "uphill point," and the remaining $20 \%$ predicted equal speeds at these two points.

The popularity of the slope-speed response was unaffected by the completion of a prior physics course. The response percentages for these participants and those without prior physics instruction are again listed in Appendix C. A majority of each group chose the slope-speed response. These percentages statistically exceeded chance (both $z \mathrm{~s}>2.10$, both $p \mathrm{~s}<.01$ ). In addition, the completion of a prior physics course was independent of the likelihood of choosing the belief response, $\chi^{2}(1)=<0.01, n s$, and the pointbiserial correlation between the number of physics courses and the likelihood of choosing the slope-speed response did not differ statistically from zero $\left(r_{\mathrm{pb}}=.06, n s\right)$.

The slope-speed response was popular with both men and women. The precise response percentages for each gender are listed in Appendix C. For women, the percentage of slope-speed responses was statistically greater than chance $(z=3.24, p<.01)$. For men the slope-speed response was the modal response, but the percentage exceeded chance by a statistically insignificant margin $z=0.45, n s$. Nevertheless, a chi-square test of independence revealed no significant interaction between gender and the percentage of slope-speed responses, $\chi^{2}(1)=0.99, n s$.

The written justifications provided strong support for the slopespeed belief. Of those participants who chose the slope-speed response, $63 \%$ cited the difference in slope. Another $15 \%$ cited the greater force at the "downhill point," which might reflect the contribution of the resistance-speed fallacy. Of those who correctly predicted greater speed at the "uphill point" within the dip, $92 \%$ appropriately credited either the drop downhill within the dip or the uphill section of the hill. Of those who incorrectly predicted equal speeds at both points, $40 \%$ believed that the effects of the dip and the hill were equivalent. Further details are given in Table D2 of Appendix D.

In summary, a majority of participants predicted incorrectly that incline speed at a point reflects the incline slope at that point. The prevalence of this slope-speed belief was nearly unaffected by 
either gender or a prior physics course. In addition, a majority of these slope-speed believers explicitly cited the slope-speed belief in their written justifications.

\section{Experiment 3}

This experiment tested the possibility that the prevalence of the slope-speed belief reflects the resistance-speed fallacy. As described in the introduction, this fallacy attributes incline speed to the resistance encountered as objects are lifted or pushed along inclines. Because this resistance varies with slope, the resistancespeed fallacy predicts greater speed at points of greater resistance. Hence, this fallacy holds that speed is greater during descent than during ascent, and likewise, it predicts that downhill speed is greater along steeper sections.

The participants in this experiment answered the speed question, but one half of them, those in the resistance condition (vs. the control condition), first answered a question concerning resistance. This resistance question concerned two points along a concave ramp similar to that shown in Figure 1A; the slopes at the two indicated points differed sharply. I held a sphere at each point, one at a time. Participants were asked to choose the location that required "more strength." The correct answer is the "steeper point," of course, because resistance increases with slope. Hence, this question effectively reminded or primed participants of the factual relationship between slope and resistance. Consequently, if a participant was reminded that slope predicts resistance and if the participant already believed that resistance predicts speed (i.e., the resistance-speed fallacy), the participant would be more likely to predict that slope predicts speed. Note that the resistance question did not suggest a link between resistance and speed. In fact, the question did not include a reference to either speed or motion.

Therefore, if the resistance-speed fallacy contributes to the prevalence of the slope-speed belief, the slope-speed response should be more popular among resistance participants than control participants. In effect, there should be an interaction between response and condition. Because interactions are most easily interpreted for $2 \times 2$ designs, the speed question included only two response choices. These alternatives included the correct response (the lower point) and the slope-speed response (the higher point); participants could simply infer that the speeds were not equal.

\section{Method}

Participants. A total of 50 undergraduate students from the University of South Florida participated in return for course credit. This sample included 32 women and 18 men; 32 reported that they had completed at least one physics course in high school or college. None had participated in Experiments 1 or 2.

Materials and procedure. Participants were randomly assigned to either the resistance or the control conditions and tested in small groups. Each condition included 25 participants, and only the participants in the resistance condition answered the resistance question. All participants answered the speed question.

The resistance question used a concave ramp that was $62 \mathrm{~cm}$ long $\times 3.5$ $\mathrm{cm}$ wide. The ramp's shape and the location of the critical points $X$ and $Y$ were almost identical to those of the ramp shown in Figure 1A; the sphere's diameter was $2.5 \mathrm{~cm}$. The resistance question was read aloud as follows (and the actions in parentheses were performed): "In the first scenario, I hold the marble at Point X on the ramp (I did so). In the second scenario, I hold the marble at Point $\mathrm{Y}$ on the ramp (I did so). Which scenario requires more strength? That is, does it require more strength to hold the marble at Point X or at Point Y?" The answer sheets included the options Point $X$ and Point $Y$.

The experiment used the same apparatus and marbles as in the previous experiments, and the question was read aloud as follows (and the actions in parentheses were performed): "These two marbles are identical (I showed the marbles to participants). Marble A is placed at the start of the ramp with the dip (I placed the marble at the start of the dip ramp and pointed to the dip). Marble B is placed at the start of the ramp with the hill (I placed a second marble at the start of the hill ramp and pointed to the hill). But the marbles will not be released at the same time, because this is not a race. Instead, the marbles will be released one at a time. Your task is to predict which marble will be rolling faster at the instant it reaches this mark (I pointed to each point and the line segment adjoining the points). That is, regardless of how quickly each marble reaches this line (I pointed again), is Marble A rolling faster than Marble B at this point, or is Marble B rolling faster than Marble A at this point (I specifically indicated each critical point)?" Immediately afterward, each participant provided a brief written explanation of his or her answer.

\section{Results and Discussion}

As shown in Figure 4C, the likelihood of choosing the slopespeed response to the speed question was increased dramatically by prior exposure to the resistance question. In the resistance condition, all but one correctly answered the resistance question $(z=4.60, p<.01)$, and $76 \%$ subsequently chose the slope-speed response to the speed question $(z=2.60, p<.01)$. In the control condition, $44 \%$ chose the slope-speed response. The interaction between condition and response was statistically significant, $\chi^{2}(1)=5.33, p<.05$.

It is not clear why the slope-speed response was not the modal response in the control condition, in light of its appeal in Experiment 2 . The questions in the two experiments varied slightly, but the different outcomes probably reflect small differences in the sample and perhaps some sampling error. Yet almost half of the controls in this experiment still chose the slope-speed response.

An analysis of the participants' reasons revealed that most slope-speed responders explicitly attributed their answers to the slope-speed belief. Note that the tendency of slope-speed responders to cite the slope-speed belief was greater in the resistance condition than in the control condition. The percentages are listed in Table D3 of Appendix D. Of those who correctly predicted greater speed at the "uphill point" within the dip, most participants appropriately cited the effect of the ramp shape prior to the critical points.

In summary, the responses and the written justifications suggested that a large percentage of the participants believed falsely that speed depends on slope. In addition, the appeal of the slopespeed belief was increased sharply when participants first considered the relationship between slope and the effort one must exert to hold an object at a point on an incline. The mere consideration of this factual link between slope and resistance induced participants to later attribute incline speed to incline slope; this finding is consistent with the view that the resistance-speed fallacy contributes to the slope-speed belief. 


\section{General Discussion}

\section{Summary}

Five primary findings are presented. First, Experiment 1 provided evidence of broad support for the misguided view that the shortest incline ramp is necessarily the quickest (i.e., the shorterquicker belief). Second, Experiment 2 revealed the prevalence of the false belief attributing an object's incline speed at a given point to the incline slope at that point (i.e., the slope-speed belief). Third, neither gender nor prior physics instruction affected the appeal of either the shorter-quicker belief or the slope-speed belief. Fourth, Experiment 3 provided evidence for the view that the slope-speed belief reflects the resistance-speed fallacy. This deeper misconception attributes an object's gravity-induced speed to the amount of resistance encountered while traversing inclines or pushing objects along inclines. This resistance is greater at steeper slopes; therefore the resistance-speed fallacy explains the slope-speed belief. Fifth, it was shown theoretically that the resistance-speed fallacy also predicts the broad appeal of the previously reported mass-speed beliefs, holding that incline speed and free fall speed are greater for heavier objects than for lighter objects.

\section{Animated Images}

The appeal of the slope-speed belief might be reduced dramatically if participants observed its simulation. For example, rather than predict the outcome of a scenario like those presented in the experiments presented here, participants could choose from among multiple animated sequences. One animation would obey the laws of Newtonian theory, and another would abide by the slope-speed belief. Consequently, participants who predict the slope-speed response for a particular scenario might be able to reject the slope-speed response after observing its animation. Indeed, reversals of this kind have been observed in studies using animations of previously reported misconceptions about motion (e.g., Kaiser, Proffitt, \& Anderson, 1985). Yet, intriguingly, some false beliefs remain appealing even when animated (see, e.g., Hecht \& Bertamini, 2000). Kaiser, Proffitt, Whelan, and Hecht (1992) reviewed the influence of animation on a variety of false beliefs and concluded that animation evokes the appropriate view when the motion can be characterized by a single parameter. In this account, an animated version of the slope-speed belief should appear unnatural, because variation in rolling incline speed depends almost solely on vertical drop.

\section{The Peaceful Coexistence of Truth and False Belief}

In this study, the appeal of the false beliefs was unaffected by prior instruction in physics. Of course, physics instruction was not a manipulated variable, and therefore the observed null effects of physics training are subject to the usual caveats (cf. Donley \& Ashcraft, 1992). Yet if it is assumed that the mechanical aptitude of students who complete a physics course is at least as great as those who do not, the results reported here are certainly troubling for educators. Similar findings have led some educators to suggest supplementing the physics curricula with animations of various misconceptions, and these kinds of demonstrations might reduce the appeal of the shorter-quicker and slope-speed beliefs. Note, however, that these interventions have had mixed results (cf. Kaiser et al., 1992).

Despite the futility of prior physics instruction in the present experiments, it remains unknown as to whether these false beliefs would prove appealing to those who possess expertise in incline motion. These experts might include formally trained physicists as well as those who have considerable exposure to incline motion. Yet previous studies have revealed that such expertise does not always provide immunity. For example, Proffitt et al. (1990) found that neither physics teachers nor bicyclists possessed a basic understanding of wheel dynamics. More recently, Hecht and Proffitt (1995) reported that bartenders performed worse than a matched control group on the Piagetian water-level task (which concerns liquids in cylindrical containers). These kinds of results are nominally contradictory, because it appears that the appeal of the false belief is unaffected by newly acquired expertise.

The paradox is resolved, of course, by assuming that the truth and the false belief can peacefully coexist. In this account, a single individual holds multiple beliefs-some appropriate and some not-and the relative appeal of each depends on the context and precise nature of a given scenario. Indeed, a number of studies have found that a single individual may succumb to a false belief in one instance but prove immune in a similar scenario (cf. Cooke \& Breedin, 1994; Kaiser, Jonides, \& Alexander, 1986; Proffitt \& Gilden, 1989). Likewise, the participants who fell prey to the slope-speed belief in this study might easily reject it in another. For instance, if these students were asked to compare incline speed at two points along a linear incline, it is doubtful that more than a few would predict equal speeds at two points of equal slope (as necessitated by the slope-speed belief). Hence, the findings reported here do not suggest that people fail to appreciate the relationship between incline speed and vertical drop.

In the same line of reasoning, those participants who correctly predicted the outcome of the speed question might succumb to the slope-speed belief in another context. Thus, like any behavior, dynamical judgments depend on the nature of the stimulus. In this view, the false beliefs reported here should not be construed as the beliefs of only some people but rather as universal beliefs that are suppressed with varying degrees of success.

More broadly, then, people appear to choose from a set of heuristics that provide efficiency at the expense of occasional errors (cf. Proffitt \& Gilden, 1989). For instance, Pittenger (1991) observed that although people appear to falsely believe that the period of a pendulum is proportional to its length, this belief is generally sufficient. Likewise, the shorter-quicker belief is a simple heuristic; the downhill skier who chooses the shortest path down the mountain has usually chosen the quickest path as well. In a similar vein, Abernathy (1993) argued that the visual system uses the least amount of information that is necessary to accomplish a task, and Braunstein (1994) posited the existence of heuristic processes to explain people's perception of three-dimensional objects from two-dimensional images. Moreover, people's reliance on heuristics is not always by choice, because the relevant algorithm may not be solvable. For this reason, Hecht (2000) concluded that these heuristics are sometimes necessary, and he further invoked the notion of "satisficing," to explain that the selection of these heuristics can vary in response to people's motivations, cognitions, and emotions. 


\section{The Resistance-Speed Fallacy}

The possibility that the resistance-speed fallacy underlies the mass-speed beliefs could be tested in a future line of research. For example, participants might predict the outcome of a race between objects of different mass along two identical inclines, with one half of the participants actually placing the objects at the start of the ramp. If these participants pushed the light object and heavy object up the incline before predicting the outcome of the race, the salient difference in the objects' resistances might increase the appeal of the mass-speed belief. In an analogous free fall experiment, half of the participants would lift the heavy and light objects before predicting the outcome of the race to the floor. These experiments simply prime the link between mass and resistance, just as the resistance question in Experiment 3 primed the association between slope and resistance. For either proposed experiment, an increase in the percentage of mass-speed responses by the actively engaged participants would provide evidence for the view that the mass-speed beliefs reflect the resistance-speed fallacy. These proposed experiments rely on the salience of an object's mass, and this salience can have large effects on people's judgments. In an experiment reported by Bhalla and Proffit (1999), for example, college students who stood at the base of a hill provided greater visual estimates of the hill's slope when they were burdened by a moderately heavy backpack.

Note that the resistance-speed fallacy cannot be corrected by simply replacing the term speed with the term acceleration. In fact, the amount of resistance encountered before the object's release may or may not be associated with its rate of acceleration after its release. For instance, if two identical marbles are raced along inclines of different slope, both their resistances prior to release and their subsequent accelerations differ. In contrast, if two objects of unequal mass are placed atop identical inclines and then released, the two objects provide different amounts of resistance before release and equal rates of acceleration after release. In summary, encountered resistance predicts neither speed nor acceleration, and the resistance-speed fallacy is not simply another example of the widely observed confusion between speed and acceleration.

Furthermore, the resistance-speed fallacy is not predicted by the popular pre-Newtonian theories of motion. For instance, false beliefs about vertical free fall are sometimes consistent with the so-called Aristotelian error that mistakenly relates force to speed instead of to acceleration (e.g., Clement, 1982; Shanon, 1976). Other common misconceptions are predicted by a pre-Newtonian system of beliefs known as impetus theory, which allows an object to change its speed or direction in the absence of net force. For example, when people are asked to predict the path of a marble after it exits a spiral tube placed atop a flat table, many predict that the marble's path will initially curve before straightening out (cf. Kaiser, Jonides, \& Alexander, 1986; Kaiser, McCloskey, \& Proffitt, 1986; Kaiser et al., 1985; McCloskey, Caramazza, \& Green, 1980; McCloskey \& Kohl, 1983). Although these pre-Newtonian false beliefs have certainly contributed to researchers' understanding of dynamical judgments, the beliefs' uses are arguably unrelated to their links with pre-Newtonian theories of motion.

The resistance-speed fallacy is, however, consistent with accounts that attribute people's failures of dynamical judgments to their understanding of the laws governing their interaction with objects. For instance, Hecht and Bertamini (2000) found that a large percentage of an adult sample falsely believed that an upwardly thrown object continues to accelerate after it leaves the thrower's hand; this false belief may reflect that the thrower must accelerate the object before it leaves the hand. In effect, the dynamics of the critically important interactive phase are falsely extrapolated to the subsequent trajectory. Hecht and Bertamini described this as an externalization of people's body dynamics. Likewise, people cannot push objects uphill or lift objects from the ground without calibrating their efforts in response to the encountered resistance. This most-salient aspect of the critical interactive phase may disrupt people's understanding of the subsequent motion, which can be ignored without consequence.

\section{References}

Abernethy, B. (1993). Searching for the minimal essential information for skilled perception and action. Psychological Research, 55, 131-138.

Anton, H. (1980). Calculus with analytic geometry (2nd ed.). New York: Wiley.

Bell, E. T. (1937). Men of mathematics. New York: Simon \& Schuster.

Bhalla, M., \& Proffitt, D. R. (1999). Visual-motor recalibration in geographical slant perception. Journal of Experimental Psychology: Human Perception and Performance, 25, 1076-1096.

Braunstein, M. L. (1994). Decoding principles, heuristics, and inferences in visual perception. In G. Jansson, S. S. Bergström, \& W. Epstein (Eds.), Perceiving events and objects (pp. 436-446). Hillsdale, NJ: Erlbaum.

Clement, J. (1982). Students' preconceptions in introductory mechanics. American Journal of Physics, 50, 66-71.

Cooke, N. J., \& Breedin, S. D. (1994). Constructing naive theories of motion on the fly. Memory \& Cognition, 22, 474-493.

Crawford, F. S. (1996). Rolling and slipping down Galileo's plane: Rhythm of the spheres. American Journal of Physics, 64, 541-546.

Creem, S. H., \& Proffitt, D. R. (1998). Two memories for geographical slant: Separation and interdependence of action and awareness. Psychonomic Bulletin \& Review, 5, 22-36.

Donley, R. D., \& Ashcraft, M. H. (1992). The methodology of testing naïve beliefs in the physics classroom. Memory \& Cognition, 20, 381-391.

Drake, S. (1989). Galileo's gravitational units. The Physics Teacher, 27, 432-436.

Halliday, D., \& Resnick. R. (1981). Fundamentals of physics (2nd ed., extended). New York: Wiley.

Hecht, H. (1993). Judging rolling wheels: Dynamic and kinematic aspects of rotation-translation coupling. Perception, 22, 917-928.

Hecht, H. (2000). The failings of three event perception theories. Journal for the Theory of Social Behaviour, 30, 1-25.

Hecht, H., \& Bertamini, M. (2000). Understanding projectile acceleration. Journal of Experimental Psychology: Human Perception and Performance, 26, 730-746.

Hecht, H., \& Proffitt, D. R. (1995). The price of expertise: Effects of experience on the water-level task. Psychological Science, 6, 90-95.

Kaiser, M. K., Jonides, J., \& Alexander, J. (1986). Intuitive reasoning about abstract and familiar physics problems. Memory \& Cognition, 14, $308-312$.

Kaiser, M. K., McCloskey, M., \& Proffitt, D. R. (1986). Development of intuitive theories of motion: Curvilinear motion in the absence of external forces. Developmental Psychology, 22, 67-71.

Kaiser, M. K., Proffitt, D. R., \& Anderson, K. (1985). Judgments of natural and anomalous trajectories in the presence and absence of motion. Journal of Experimental Psychology: Learning, Memory, and Cognition, 11, 795-803.

Kaiser, M. K., Proffitt, D. R., Whelan, S. M, \& Hecht, H. (1992). Influence of animation on dynamical judgments. Journal of Experimental Psychology: Human Perception and Performance, 8, 669-689. 
Krist, H., Fieberg, E. L., \& Wilkening, F. (1993). Intuitive physics in action and judgment: The development of knowledge about projectile motion. Journal of Experimental Psychology: Learning, Memory, and Cognition, 19, 952-966.

McBeath, M. K., Shaffer, D. M., \& Kaiser, M. K. (1995, April 28). How baseball outfielders determine where to run to catch fly balls. Science, 268, 569-573.

McCloskey, M., Caramazza, A., \& Green, B. (1980, December 5). Curvilinear motion in the absence of external forces: Naive beliefs about the motion of objects. Science, 210, 1139-1141.

McCloskey, M., \& Kohl, D. (1983). Naive physics: The curvilinear impetus principle and its role in interactions with moving objects. Journal of Experimental Psychology: Learning, Memory, and Cognition, 9, 146156

Pittenger, J. B. (1985). Estimation of pendulum length from information in motion. Perception, 14, 247-256.
Pittenger, J. B. (1991). Cognitive physics and event perception: Two approaches to the assessment of people's knowledge of physics. In R. R. Hoffman \& D. S. Palermo (Eds.), Cognition and the symbolic process: Applied and ecological perspectives (pp. 233-254). Hillsdale, NJ: Erlbaum.

Proffitt, D. R., Bhalla, M., Gossweiler, R., \& Midgett, J. (1995). Perceiving geographical slant. Psychonomic Bulletin \& Review, 2, 409-428.

Proffitt, D. R., \& Gilden, D. L. (1989). Understanding natural dynamics. Journal of Experimental Psychology: Human Perception and Performance, 15, 384-393.

Proffitt, D. R., Kaiser, M. K., \& Whelan, S. M. (1990). Understanding wheel dynamics. Cognitive Psychology, 22, 342-373.

Rohrer, D. (1994). More thought provokers. Berkeley, CA: Key Curriculum Press.

Shanon, B. (1976). Aristotelianism, Newtonianism and the physics of the layman. Perception, 5, 241-243.

\section{Appendix A}

\section{Ramp Specifications}

The precise specifications of the ramp shape are illustrated by the Cartesian plot in Figure 2B. In the figure, each grid square represents a square with sides of $10 \mathrm{~cm}$, and consequently, each ramp provided a net vertical drop of $10 \mathrm{~cm}$ and a horizontal displacement of $80 \mathrm{~cm}$. Each ramp comprised two horizontal line segments and four arcs, and each of these six sections spanned exactly one or two horizontal units of the grid. With respect to this grid in Figure 2B, the six sections are defined as follows (lengths are in centimeters):

1. For $x=[0,20]$, there is a $53.13^{\circ}$ arc with radius 25 and center $(20,25)$

2. For $x=[20,30]$, there is a horizontal line segment on the $y$-axis.
3. For $x=[30,40]$, there is a $30^{\circ}$ arc with radius 20 and center $(30,-20)$.

4. For $x=[40,60]$, there is a $60^{\circ}$ arc with radius 20 and center $(50,14.6)$.

5. For $x=[60,70]$, there is a $30^{\circ}$ arc with radius 20 and center $(70,-20)$.

6 . For $x=[70,80]$, there is a horizontal line segment on the $y$-axis.

For the speed question, the two comparison points had an $x$-coordinate of 60 , and each was a point of inflection (i.e., the second derivative equaled $0)$. It is important to note that each ramp shape was everywhere differentiable, thereby ensuring that any two consecutive sections had the same slope at the point connecting the sections. This eliminated cusps and therefore produced a smooth roll.

\section{Appendix B}

\section{Newtonian Mechanics of Incline Motion}

This appendix describes some of the Newtonian theory governing incline motion. The presentation is conceptual, and the mathematical details are given in most introductory physics textbooks.

Velocity is represented by a vector with magnitude and direction. This vector is itself a vector sum of its horizontal and vertical components. Acceleration equals the change in velocity, and acceleration can therefore reflect a change in either speed or direction. An object accelerates if and only if it is under the influence of a nonzero net force, as given by Newton's second law (Force $=$ mass $\times$ acceleration).

An object on an incline is under the influence of both the gravitational force and the normal force. The gravitational force is directed downward, and the incline exerts the normal force in a direction that is perpendicular to the incline surface. Hence, along horizontal ramps, the normal force is directed upward. Along descents, the normal force is directed upward and forward. Along ascents, the normal force is directed upward and backward.

The vector sum of the gravitational force and the normal force equals the net force producing incline descent. This net force can be conceptualized as the vector sum of its vertical and horizontal components. Because the gravitational force is directed downward, the horizontal component of the net force is equal to the horizontal component of the normal force. 


\section{Appendix C}

Response Percentages by Gender and Physics Background

\begin{tabular}{ccc}
\hline Participants and responses & $\begin{array}{c}\text { Experiment 1: } \\
\text { Race question }\end{array}$ & $\begin{array}{c}\text { Experiment 2: } \\
\text { Speed question }\end{array}$ \\
\hline Overall & & \\
Belief & 60 & 54 \\
Truth & 22 & 26 \\
Other & 18 & 20 \\
Physics & & \\
Belief & 61 & 55 \\
Truth & 21 & 27 \\
Other & 18 & 18 \\
No physics & & 54 \\
Belief & 59 & 25 \\
Truth & 23 & 21 \\
Other & 18 & \\
Women & & 58 \\
Belief & 53 & 25 \\
Truth & 28 & 18 \\
Other & 19 & 40 \\
Men & & 30 \\
Belief & 79 & 30 \\
Truth & 7 & \\
Other & 14 & \\
\hline
\end{tabular}

Note. Values in italics indicate percentages that are statistically greater than chance $(p<.01)$.

\section{Appendix D}

Participants' Reasons for Answers to Questions in Experiments 1-3

Table D1

Classification of Reasons for Answers to the Race Question: Experiment 1

\begin{tabular}{lccc}
\hline & \multicolumn{3}{c}{ Answers } \\
\cline { 2 - 4 } \multicolumn{1}{c}{ Reason } & $\begin{array}{c}\text { Shorter-quicker belief: } \\
\text { Tie (\%) }\end{array}$ & $\begin{array}{c}\text { Truth: } \\
\text { Dip (\%) }\end{array}$ & $\begin{array}{c}\text { Other: } \\
\text { Hill (\%) }\end{array}$ \\
\hline Ramps have equal length & 27 & \\
Ramps are symmetric & 50 & 27 \\
Dip increases speed & & 18 & \\
Hill slows speed & & 36 & 44 \\
Downhill part of dip increases speed & & & 33 \\
Ramp is quicker when incline precedes decline & & 18 & 22 \\
Marble on dip ramp will not escape dip & 23 & & \\
Other & & & \\
\hline
\end{tabular}


Table D2

Classification of Reasons for Answers to the Speed Question: Experiment 2

\begin{tabular}{lccc}
\hline \multicolumn{1}{c}{ Reason } & \multicolumn{3}{c}{ Answers } \\
\cline { 2 - 4 } & $\begin{array}{c}\text { Slope-speed belief: } \\
\text { Downhill (\%) }\end{array}$ & $\begin{array}{c}\text { Truth: } \\
\text { Uphill (\%) }\end{array}$ & $\begin{array}{c}\text { Other: } \\
\text { Equal (\%) }\end{array}$ \\
\hline $\begin{array}{l}\text { Difference in slope } \\
\text { Greater force or gravity at downhill point }\end{array}$ & 63 & & \\
$\begin{array}{l}\text { Speed increased by drop downhill within the dip } \\
\text { Speed decreased by uphill section within the hill }\end{array}$ & 15 & 84 & \\
Effects of the dip and hill exactly cancel & & 8 & 40 \\
Speed at point depends on prior distance & & & 10 \\
Other & 22 & 8 & 50 \\
\hline
\end{tabular}

Table D3

Classification of Reasons for Answers to the Two-Alternative Speed Question: Experiment 3

\begin{tabular}{|c|c|c|c|c|}
\hline \multirow[b]{3}{*}{ Reason } & \multicolumn{4}{|c|}{ Answers } \\
\hline & \multicolumn{2}{|c|}{ Slope-speed belief: Downhill } & \multicolumn{2}{|c|}{ Truth: Uphill } \\
\hline & Resistance (\%) & Control (\%) & Resistance (\%) & Control $(\%)$ \\
\hline Slope & 95 & 64 & & \\
\hline Dip increases speed & & & 33 & 64 \\
\hline Hill decreases speed & & & 17 & 7 \\
\hline Difference in elevation & & & 17 & 7 \\
\hline Other & 5 & 36 & 33 & 21 \\
\hline
\end{tabular}

Received February 7, 2001

Revision received November 13, 2001

Accepted January 9, 2002 